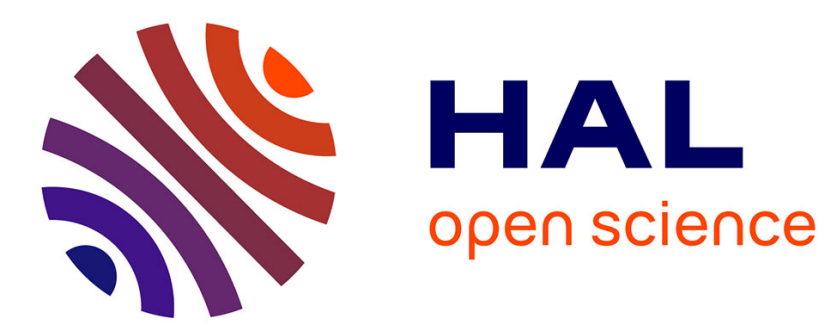

\title{
Représentation logique et spatiale de la réglementation des activités humaines en zone côtière
}

\author{
Matthieu Le Tixerant
}

\section{To cite this version:}

Matthieu Le Tixerant. Représentation logique et spatiale de la réglementation des activités humaines en zone côtière. Revue Internationale de Géomatique, 2002, Volume 12 (3/2002), p. 325 à 335. hal-01009731

\section{HAL Id: hal-01009731 \\ https://hal.science/hal-01009731}

Submitted on 19 Jun 2014

HAL is a multi-disciplinary open access archive for the deposit and dissemination of scientific research documents, whether they are published or not. The documents may come from teaching and research institutions in France or abroad, or from public or private research centers.
L'archive ouverte pluridisciplinaire HAL, est destinée au dépôt et à la diffusion de documents scientifiques de niveau recherche, publiés ou non, émanant des établissements d'enseignement et de recherche français ou étrangers, des laboratoires publics ou privés. 


\title{
Représentation logique et spatiale de la réglementation des activités humaines en zone côtière.
}

\author{
Matthieu Le Tixerant \\ Doctorant au laboratoire Géosystèmes, UMR 6554 CNRS, Institut Universitaire \\ Européen de la Mer, Université de Bretagne Occidentale. Place Nicolas Copernic, \\ 29280 Plouzané \\ Matthieu.Letixerant@univ-brest.fr
}

\begin{abstract}
RESUME. La mesure de l'impact des usages anthropiques sur l'environnement est un enjeu majeur du développement durable des zones côtières. Cela implique avant tout de pouvoir décrire et comprendre le déroulement et les modalités de l'organisation des activités humaines ; d'où la nécessité de développer des outils décrivant la dynamique des processus qui leur sont associés. Il est donc fondamentale, et cela est particulièrement vrai en milieu marin, de pouvoir accéder rapidement aux contraintes réglementaires et d'analyser leur articulation dans l'espace et dans le temps. Une méthode de structuration logique de la réglementation appliquée au cas de la pêche en mer d'Iroise est proposée dans un objectif opérationnel de gestion des usages. Son intégration au sein d'un Système d'Information Géographique (SIG) produit des représentations spatiales de manière automatisée et à partir de requêtes variées.
\end{abstract}

ABSTRACT. The measurement of anthropic uses impact on environnement is a major stake of sustainable coastal zones management. That implies to be able to describe and understand the progress and organisation of present human activities. In fact we need to develop tools allowing a dynamic description of the processes related to the coastal zone human activities. In this way, and this is particularly true in a marine area, it is indeed fundamental to be able to reach quickly the lawful constraints and analyse their space and time articulations. Applied to the case of fishing in the Iroise sea, a method to carry out a logical regulation structuring is proposed. Its integration within a Geographic Information System (GIS) can produce space representations in an automated way and according to varied requests.

MOTS-CLES : Zones côtières, activités humaines, réglementation, structuration logique, SIG, représentation spatiale.

KEYWORDS : coastals zones, human activities, regulation, logic structuration, GIS, spatial representation

Revue Internationale de Géomatique 


\section{Introduction}

La gestion intégrée des zones côtières doit prendre en compte les changements à long terme de l'environnement sous la double influence des variations climatiques et de l'évolution des sociétés humaines [HOL]. La singularité de la zone côtière par rapport aux espaces continentaux tient à sa position unique à l'interface entre l'atmosphère, la terre et l'océan. Cette situation est à l'origine de milieux complexes et très spécifiques qui ont généré de multiples usages. L'action de l'Homme est donc bien au cœur du système. Elle se traduit par l'exploitation des ressources naturelles et par l'aménagement des espaces, ce qui est susceptible d'entraîner des perturbations importantes sur les conditions d'équilibre des milieux.

L'analyse des interactions entre les activités humaines et l'environnement est l'un des principaux objectifs de la recherche pour le développement durable des zones côtières [CUQ 00]. Cette démarche se fonde sur une bonne description et compréhension du déroulement et des modalités d'organisation des activités humaines, avant d'aborder la question complexe de leur impact sur le milieu. Il est fondamental de pouvoir accéder aux contraintes réglementaires et d'analyser leurs répercussions sur l'espace et dans le temps. Il semble en effet difficile de comprendre le fonctionnement du système si l'on fait abstraction du cadre réglementaire dans lequel il s'inscrit. La réglementation encadre les activités notamment en terme d'extension géographique, de période et d'intensité. Sur un « espace ouvert» tel que le domaine marin, elle constitue donc un premier « filtre » pour tenter d'identifier les zones et périodes concernées par le déroulement d'une activité. Cela est particulièrement vrai pour des activités telles que la pêche côtière qui est très difficile à appréhender, en raison de la grande dispersion des flottilles le long du littoral [BOL 00]. Pour prendre des décisions pertinentes, les gestionnaires doivent pouvoir accéder rapidement à des informations utiles et adéquates [COM 00 ] et comprendre où et comment s'applique le droit sur leur espace de compétence. Dans ce contexte, l'intégration de données juridiques au sein d'un SIG peut s'avérer un élément d'analyse et de connaissance très utile.

Cet article a donc pour objectif de présenter une méthode permettant :

- de dresser un inventaire de la réglementation en zone côtière et de la structurer dans la perspective d'une gestion intégrée.

- d'opérer une représentation logique de la réglementation intégrable au sein d'un SIG afin de réaliser des cartes de manière automatisée.

En conclusion sont discutées les perspectives offertes par cette démarche et l'intérêt de son élargissement pour concevoir un modèle des usages en zone côtière. 


\section{Le cadre de l'étude}

Située à l'extrémité occidentale du Finistère, la mer d'Iroise constitue un écosystème marin d'une grande richesse. De multiples usages et activités potentiellement conflictuels, s'y côtoient et fragilisent son équilibre. Seule une gestion intégrée de l'ensemble de la zone permettrait d'assurer son développement durable. Depuis une dizaine d'années, l'Etat favorise donc l'élaboration d'un projet de parc national marin. Les études scientifiques réalisées mettent notamment en évidence la nécessité de mieux lier les activités et les réglementations qui les structurent aux espaces sur lesquels ils s'appliquent. Dans ce contexte, le recensement, la structuration et la représentation des espaces réglementés en mer d'Iroise ont été réalisés en collaboration étroite avec la Mission pour la création du Parc National Marin.

\section{Inventaire et structuration de la réglementation}

\subsection{Inventaire}

L'objectif de cet inventaire est de recenser les contraintes réglementaires qui s'exercent sur la zone et de les présenter de manière concise. Dans un premier temps, les organismes et administrations susceptibles de produire de la réglementation ont été identifiés. Ce recensement est déjà une tâche complexe car, en matière de pêche notamment, les autorités compétentes sont multiples [BEC 91]. $\mathrm{Si}$ la réglementation de la pêche est pour une large part de compétence communautaire, elle n'est pas selon le principe de subsidiarité, exclusive d'une réglementation nationale et locale.

Une approche juridique plus poussée aurait pu être envisagée. En effet, la jurisprudence, qui n'a pas pu être prise en considération dans le cadre de cette étude, peut jouer un rôle important. Une collaboration étroite avec des juristes est alors indispensable. Dans le cadre d'un suivi sur une longue période, il conviendrait de mettre en place une veille juridique constante de manière à pouvoir disposer d'une information complète et régulièrement actualisée.

Il nous a semblé préférable de travailler à partir des textes juridiques originaux. Ils constituent l'information factuelle qui peut alors être sélectionnée en connaissance de cause, contrairement aux documents de synthèse qui sont souvent entachés d'interprétation voire de subjectivité.

\section{2 la sélection de l'information}

Compte tenu de la complexité et l'importance de la réglementation des pêches en mer d'Iroise, l'exhaustivité est un objectif difficile à atteindre. Pour présenter une vision synthétique du résultat, des choix et des priorités entre les informations ont dû être établis. 
La réglementation susceptible de servir dans un contexte de gestion opérationnelle d'une aire marine protégée a été retenue en priorité. Ainsi, les textes définissant des zones et périodes de pêche interdites ou restreintes ont été privilégiés. A l'intérieur de ces zones, les réglementations visant à maintenir la ressource et à prévenir les conflits entre métiers ont également été répertoriées. En revanche, les mesures d'ordres et de police générale ainsi que les cas les plus atypiques n'ont pas été pris en considération.

\section{3 la structuration de l'information}

Une classification des données est nécessaire pour permettre de mieux comprendre la structure et le fonctionnement de l'activité. Dans le système adopté, la réglementation est classée par activité et métier suivant une typologie hiérarchique en différents niveaux: du général au spécifique (figure 1). A chaque niveau correspond une réglementation qui concerne l'activité considérée mais également ses différentes sous-activités.

\begin{tabular}{|c|c|c|c|c|c|}
\hline Niveau 0 & Niveau 1 & Niveau 2 & Niveau 3 & Niveau 4 & Niveau 5 \\
\hline \multirow{15}{*}{$\begin{array}{l}\text { Pêche } \\
\text { maritime }\end{array}$} & \multirow{15}{*}{$\begin{array}{l}\text { Autorités } \\
\text { compétentes }\end{array}$} & \multirow{12}{*}{$\begin{array}{c}\text { Pêche } \\
\text { pro. }\end{array}$} & \multirow{3}{*}{$\begin{array}{c}\text { Arts } \\
\text { trainants }\end{array}$} & Chaluts & $\begin{array}{l}\text { De Fond } \\
\text { Pélagique }\end{array}$ \\
\hline & & & & Dragues & $\begin{array}{l}\text { Coquilles } \mathrm{St} \text { Jacques } \\
\text { Autres coquillages }\end{array}$ \\
\hline & & & & Lignes & A poissons \\
\hline & & & \multirow{3}{*}{$\begin{array}{c}\text { Arts } \\
\text { dormants }\end{array}$} & Filets & \begin{tabular}{|l} 
Calés \\
Tourmants \\
Dérivants \\
\end{tabular} \\
\hline & & & & Casiers & $\begin{array}{l}\text { A crustacés } \\
\text { A mollusques }\end{array}$ \\
\hline & & & & Palangres & A poissons \\
\hline & & & \multirow{2}{*}{ Goémon } & Scoubidou & L. digitata \\
\hline & & & & Peigne & L. hyperboa \\
\hline & & & \multirow[t]{2}{*}{$\begin{array}{c}\text { Autres } \\
\text { activités }\end{array}$} & A pied & $\begin{array}{l}\text { Coguillages } \\
\text { Goémons de rive et } \\
\text { épave }\end{array}$ \\
\hline & & & & SS-marine & Ormeaux \\
\hline & & & \multirow{2}{*}{$-\begin{array}{l}\text { Cultures } \\
\text { marines }\end{array}$} & Pisiculture & \\
\hline & & & & Conchyliculture & \\
\hline & & \multirow{3}{*}{$\begin{array}{l}\text { Pêche } \\
\text { de loisir }\end{array}$} & & SS-marine & \\
\hline & & & & A pied & \\
\hline & & & & Embarquée & \\
\hline
\end{tabular}

Figure 1. Structuration hiérarchique : du général au spécifique 
Pour faciliter l'utilisation opérationnelle de cette classification, nous avons procédé à une désagrégation des données. Une série de "mots clés» a été définie pour qualifier la réglementation en fonction de sa finalité. En effet la réglementation peut être relative à des entités spatiales, à des périodes, à des techniques de pêche (type d'engins, maillage des filets...), aux caractéristiques des espèces cibles (tailles minimales, quotas), ou à la nécessité de disposer d'une autorisation administrative (licence...). Ainsi dans le cas d'une interdiction de pêche sur une zone, la variable significative ou mot clé approprié est « zonage ». Cette première phase d'analyse et de structuration des données permet un accès rapide aux contraintes réglementaires grâce à une présentation synthétique sous forme de tableaux (tableau 1).

\begin{tabular}{|c|c|c|c|c|}
\hline Niveau 5 & $\begin{array}{c}\text { Niveau } 6 \\
\text { « mots clés » }\end{array}$ & $\begin{array}{c}\text { Eléments constitutifs de la } \\
\text { réglementation }\end{array}$ & Textes & Sanctions \\
\hline \multirow[t]{3}{*}{$\begin{array}{l}\text { Chalut } \\
\text { Pélagique }\end{array}$} & Zonage & $\begin{array}{l}\begin{array}{l}\text { Pêche interdite sur les plateaux } \\
\text { rocheux }\end{array} \\
\end{array}$ & $\begin{array}{l}\text { AD 152 du } \\
02 / 11 / 78 \\
\text { Art. } 4\end{array}$ & $\begin{array}{l}\text { DL du } \\
09 / 01 / 1852\end{array}$ \\
\hline & Autorisation & $\begin{array}{l}\text { Les patrons de navires désirant } \\
\text { pratiquer la pêche au chalut } \\
\text { pélagique dans les eaux } \\
\text { territoriales atlantiques doivent } \\
\text { être munis d'autorisations. }\end{array}$ & $\begin{array}{l}\text { AD 152 du } \\
02 / 11 / 78 \\
\text { Art. } 6\end{array}$ & \begin{tabular}{|l|} 
DL du \\
$09 / 01 / 1852$
\end{tabular} \\
\hline & Navire & $\begin{array}{l}\text { Pêche interdite aux navires de plus } \\
\text { de } 450 \mathrm{CV} \text { et de plus de } 50 \mathrm{TX} \text {. }\end{array}$ & $\begin{array}{l}\text { AD 152 du } \\
02 / 11 / 78 \\
\text { Art. } 2\end{array}$ & \begin{tabular}{|l} 
DL du \\
$09 / 01 / 1852$
\end{tabular} \\
\hline
\end{tabular}

Tableau 1. « mots-clés » qualifiant la réglementation du chalut pélagique

Cette organisation facilite la compréhension du cadre juridique général de l'usage par les non-spécialistes. Pour répondre à des besoins opérationnels de gestion, la compréhension des relations de construction et de composition pertinente au sein de la réglementation doit cependant être affinée. De même, la liaison des réglementations avec leur espace d'application doit être opérée. De plus, dans une optique d'intégration des données au sein d'un SIG, sa présentation doit s'adapter aux systèmes de gestion des données existants.

\section{Représentation logique et spatiale}

Pour bénéficier de l'apport potentiel des SIG en matière d'analyse spatiale et de représentation synthétique de la réglementation des usages, il est nécessaire de formaliser une méthode de conversion de l'information d'origine en une représentation logique intégrable au sein d'un Système de Gestion de Bases de Données (SGBD). Elle autorise le développement d'un système de requête adapté pour l'interrogation de la base de données. 


\subsection{Représentation logique}

En fonction des objectifs poursuivis, une représentation logique de la réglementation des différentes activités de pêche est proposée sous forme d'organigrammes. Les possibilités de réalisation de ces organigrammes sont très nombreuses et dépendent du résultat que l'on veut obtenir. Dans notre cas, l'information est structurée hiérarchiquement et suivant des critères classés selon une logique de surveillance en mer :

Structuration logique hiérarchique : conformément à la structuration établie (cf 3.3 ), les règles générales ont priorité sur les règles spécifiques : les règles communes s'appliquant à toutes les sous-activités de pêche sont d'abord prises en considération et figurent dans le haut de l'arborescence.

Structuration selon une logique de surveillance en mer : La réglementation, qualifiée par les mots-clés, est classée en fonction de son aptitude à être exploitée dans le cadre d'un contrôle par une vedette de surveillance en mer. Les contrôles qui peuvent se faire aisément depuis la vedette sont d'abord pris en considération. Ainsi les critères du «zonage » et de la «période » sont considérés comme une priorité. Il faut déjà s'assurer que le navire surveillé n'est pas en pêche sur une zone et/ou pendant une période interdite. Ensuite sont pris en compte les critères qui obligent la vedette à s'approcher des engins de pêche mouillés (pour contrôler qu'ils sont bien signalés et identifiés) et éventuellement à aborder les navires (pour vérifier qu'ils possèdent bien les autorisations nécessaires et des engins de pêche conformes).

Représentation logique du respect ou du non-respect de la réglementation : Pour chaque mot clé qualifiant une réglementation, plusieurs types de questions se posent : la pêche est-elle autorisée sur cette zone ? la pêche est-elle autorisée à cette période de l'année ? Les engins de pêche sont-ils identifiés et signalés? Les règles de maillage sont-elles respectées? Les engins utilisés sont-ils conformes? Après chaque question, deux choix sont donc possibles :

- «Oui», représenté par des traits pleins sur les organigrammes.

- «Non », représenté par les traits en pointillés sur les organigrammes.

Ainsi dans le cas du contrôle d'un navire en activité, suivant la zone et le type de pêche, il suffit de suivre «le cheminement logique» proposé par le schéma (figure 2). Si l'on aboutit à un cadre blanc (bordure pointillée), cela signifie que le navire n'est pas en règle en vertu du texte mentionné. La référence juridique établissant la sanction est mentionnée en italique. Si l'on aboutit à un «OUI», le pêcheur répond aux exigences de la réglementation en vigueur.

Cette seconde phase de structuration des données en permet une représentation logique impliquant une analyse plus poussée de la réglementation et constituant le schéma conceptuel d'un système de requête automatisé qui pourrait préfigurer le développement d'un SIG mobile dédié à la surveillance maritime. 


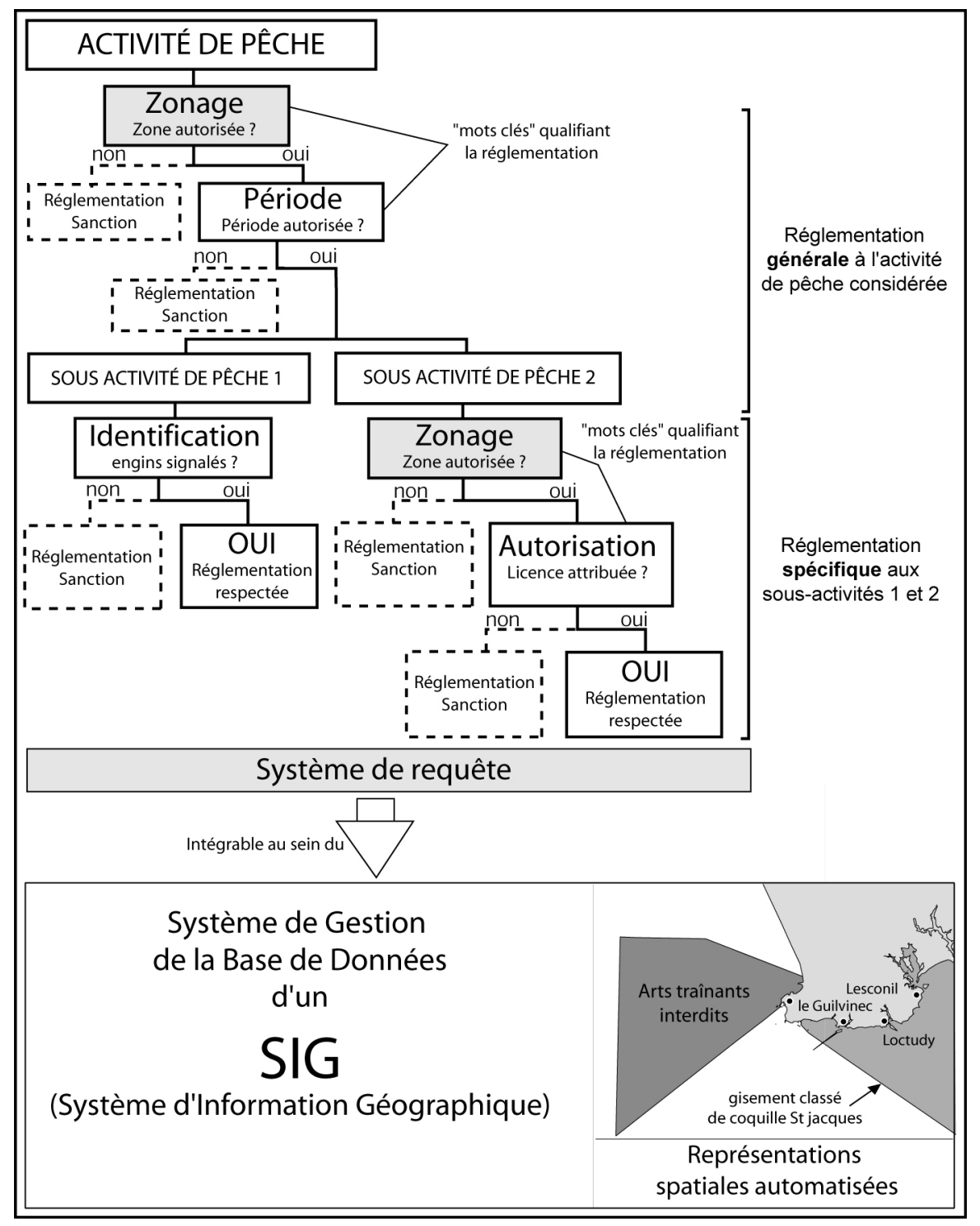

Figure 2. Principes d'une structuration logique de la réglementation intégrable au sein d'un SIG 


\subsection{Représentation spatiale}

La représentation spatiale de la réglementation des usages répond à un besoin essentiel des gestionnaires et des utilisateurs du milieu. La gestion et la prise de décision ont souvent comme support des documents cartographiques élaborés à partir d'une multitude de données spatiales. Or les SIG peuvent mobiliser sur une plate-forme unique des informations de sources variées en des temps d'accès limités, procurer une aide à la conception de cartes répondant à des besoins spécifiques (échelle, typologie...) et réduire le temps de production d'une information utile au gestionnaire par une automatisation possible des chaînes de traitement [GOU 01].

Les représentations spatiales proposées ont été réalisées avec le logiciel Arc/Info. Elles exploitent la Base d'Information Géographique (BIG) Iroise [LEB 99] qui a été enrichie pour les besoins de l'étude. Certaines zones réglementaires répertoriées au cours de la phase d'inventaire ont été numérisées afin de compléter la couche thématique «réglementation » de la BIG. Dans les textes juridiques, les différentes zones réglementées sont délimitées de différentes manières :

- par des lignes tracées à partir de différents points sur la côte (pointes, phares...), en mer (bouées, balises...) ou à partir de points définis en coordonnées géographiques (latitude / longitude),

- par l'utilisation des parallèles ou des méridiens,

- par la définition de bandes de largeur constante (bande des 3 milles) par rapport à des lignes de référence (zéro hydrographique, ligne de base droite),

- par la morphologie sous-marine (plateaux rocheux, bathymétrie).

La délimitation des zones est possible à partir de ces indications. Les différentes difficultés rencontrées ont déjà été soulignées [FOW 00] [GUI 00] [PAL 00]. Elles sont essentiellement relatives à une grande diversité et souvent imprécision des modes de délimitations utilisés au sein des textes juridiques et à l'absence de données de références communes. Pour établir un lien tangible entre la représentation logique et la représentation spatiale, les organigrammes et cartes sont représentés conjointement comme l'illustre l'exemple de la pêche au chalut en mer d'Iroise (figure 3).

Les cartes réalisées visent à fournir les éléments nécessaires à une meilleure gestion de l'environnement grâce à une compréhension fine et plus accessible de ses usages. Plus spécifiquement, elles permettent :

- de faire apparaitre de manière évidente les zones réglementées,

- la mise en évidence des interactions spatiales entre les activités qui, sur un espace ouvert tel que le domaine marin, sont susceptibles de s'exercer au même moment sur des zones identiques,

- de mieux cerner et quantifier les activités de pêche grâce à la connaissance du nombre de navires autorisés à pêcher par zone et par période. 
- d'offrir une base de travail pour mettre en évidence d'éventuelles incohérences ou redondances réglementaires et pouvoir proposer des modifications.

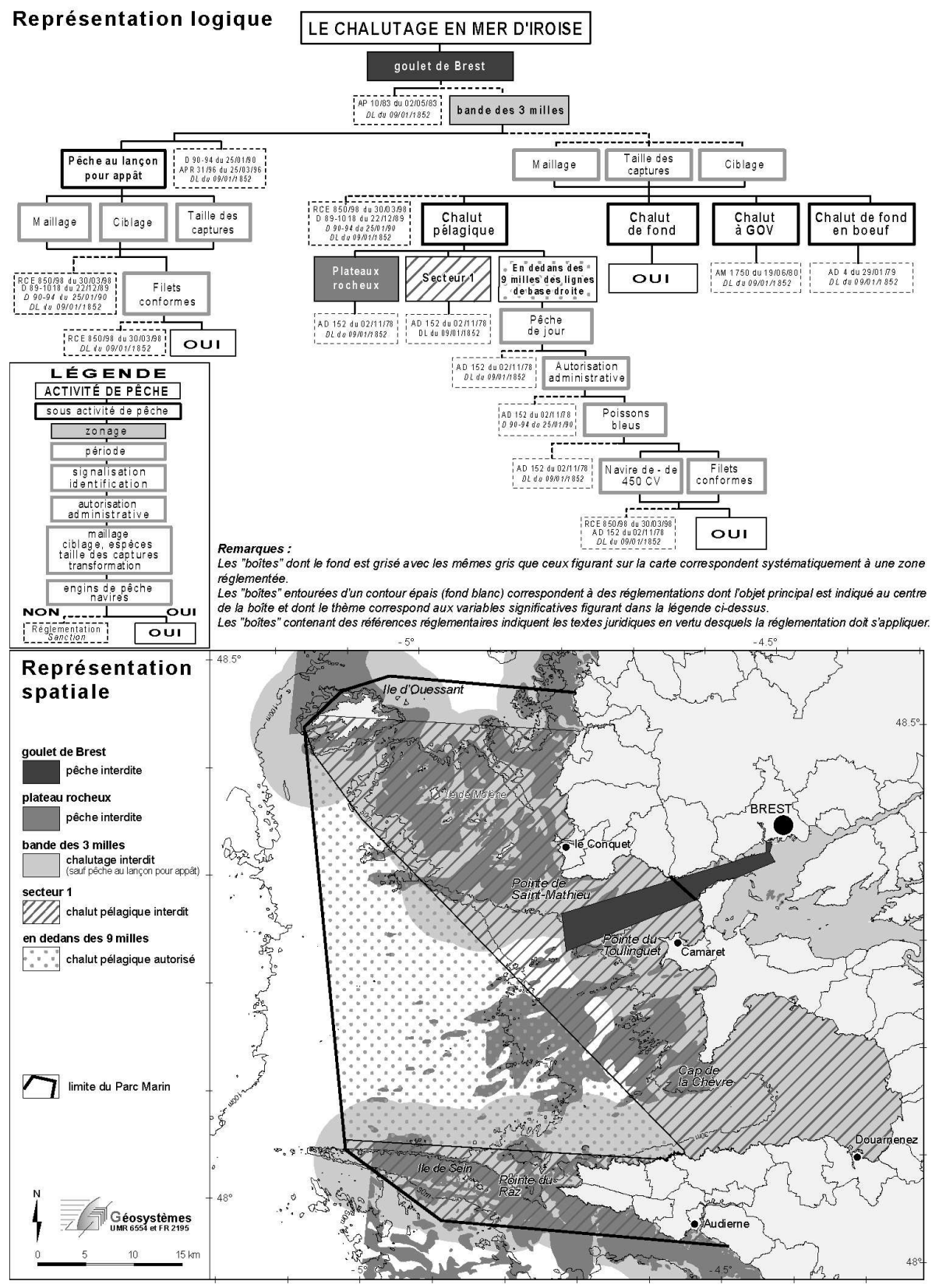

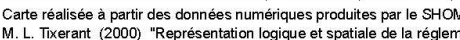

Figure 3. Représentation logique et spatiale de la pêche au chalut en mer d'Iroise 


\section{Conclusions et perspectives}

Pour développer les zones côtières de manière durable, les gestionnaires et décideurs doivent comprendre comment interagissent l'écosystème et les activités anthropiques. Dans cette contribution, l'usage du milieu est étudié sous l'angle d'une structuration logique de la représentation spatiale et de son organisation juridique. L'application traitée porte sur la réglementation des pêches en mer d'Iroise. Dans une optique de conceptualisation d'un outil informatique capable de fournir une information synthétique pouvant être utilisée dans des contextes opérationnels de gestion durable, une méthode d'intégration des contraintes réglementaires au sein d'un Système d'Information Géographique est proposée.

A l'avenir, il serait judicieux de favoriser un travail pluridisciplinaire regroupant scientifiques, acteurs et gestionnaires du milieu (Comité Régionale des Pêches, Affaires Maritimes, Préfecture Maritime) et producteurs de données (SHOM). Un premier rapport [PEN 01] visant à recenser et spatialiser les zones à accès interdit ou restreint pour la pêche professionnelle dans la bande côtière bretonne à été réalisé dans cette optique, grâce à une collaboration entre le Centre de Droit et d'Economie de la Mer et le laboratoire Géosystèmes. Ce type de partenariat devrait être poursuivi pour obtenir à terme une information régulièrement actualisée et accessible à tous (via un site Internet par exemple).

Les perspectives de travail dans ce domaine sont considérables [BAR 00]. Le besoin d'une meilleure compréhension de l'organisation générale des usages et de leurs impacts sur le milieu est de plus en plus important chez les gestionnaires. Cette étude contribue à la conception d'un système permettant de simuler et quantifier l'impact des activités humaines sur le milieu [CUQ 01]. Ce prototype devra être capable de fournir une information synthétique pouvant être utilisée dans des contextes opérationnels de gestion. Transposable à d'autres secteurs côtiers, il permettra de fournir des éléments de compréhension et d'analyse de la zone côtière grâce à une représentation spatio-temporelle du déroulement des activités humaines. Ces systèmes devront être à même de contribuer, sur un espace donné, à l'élaboration d'une politique de gestion globale en favorisant une gestion participative et une prise de conscience chez les acteurs de leur interdépendance. D'une manière plus générale il s'agit de chercher à prendre en compte la complexité de la relation entre l'Homme et son Environnement dans un objectif de développement durable. 


\section{Bibliographie}

[BAR 00] BARLETT DJ., « Working on the frontiers of science : applying GIS to the coastal zone ", in marine and coastal geographical information systems (chapter two), p. 11-24, Editions Taylor and Francis, 2000.

[BEC 91] BECET JM., LE MORVAN D., Le droit du littoral et de la mer côtière, Editions Economica, 341 p, 1991

[BOL 00] BOLOPION J., FOREST A., SOURD LJ., « Rapport sur l'exercice de la pêche dans la zone côtière de la France », rapport du Ministère de l'Agriculture et de la pêche, 107 p, janvier 2000.

[COM 00] COMMISSION EUROPEENNE «Les enseignements du programme de démonstration de la Commission européenne sur l'aménagement intégré des zones côtières ", rapport de synthèse, 97 p, 2000.

[CUQ 00] CUQ F., «Systèmes d'information Géographique et gestion intégrée des zones côtières ", Actes de Coast GIS'99: Géomatics et environnement (2000), Brest (France), Editions IFREMER, SHOM, vol 25, pp. 18-29.

[CUQ 01] CUQ F., « Analyse des interactions entre les actions humaines et le milieu littoral : une approche discrète de l'impact des activités anthropiques ", Actes de Coast GIS'01, Halifax, 9 p, 2001.

[FOW 00] FOWLER C., TREML E., HAMILTON S., « Geo-referencing the legal framework for a web-based regional ocean management geographic information system », Actes de Coast GIS'99, Brest, Editions IUEM-UBO, IFREMER, SHOM, p. 284-303 , 2000.

[GUI 00] GUILlaUMONT B., DURAND C., «Intégration et gestion de données réglementaires dans un SIG : Analyse appliquée au cas des côtes françaises », Actes de Coast GIS'99, Brest, Editions IUEM-UBO IFREMER SHOM, p 269-283, 2000.

[GOU 01] GOURMELON F., LE BERRE I., «De l'application scientifique au SIG institutionnel ; le cas du SIGIroise», Actes de CoastGIS'01, Halifax,11 p, 2001.

[HOL 94] HOLLIGAN P.M., Land Ocean Interaction In the Coastal Zone (LOICZ) : Implementation Plan. Science Plan. , IGBP: 215 p., 1994.

[LEB 99] LE BERRE I., Mise au point de méthodes d'analyse et de représentation des intéractions complexes en milieu littoral, Thèse de doctorat de géographie, Brest, UBO, 1999, $230 \mathrm{p}$.

[PAL 00] PALMER H., LORIN P., « GIS applications to maritime boundary delimitation » in marine and coastal geographical information systems, chapter two p. 11-24, Editions Taylor and Francis, 2000.

[PEN 01] PENNANGUER S., LE TIXERANT M., BONCOEUR J., «Zones à accès interdit ou restreint pour la pêche professionnelle dans la bande côtière française, cadre réglementaire et représentation spatiale (région Bretagne) », rapport CEDEM / Géosystèmes, 81 p, 2001. 